\title{
Increased Lipoprotein (a) and Its Relationships with Other Parameters of Lipoprotein Metabolism in Chronic Renal Failure Treated by Hemodialysis
}

\author{
M. Ramazan YıĞitoǦLu, MD, M. Fevzi Polat, ${ }^{1} \mathrm{MSc}$, \\ Fatih AkçAY, ${ }^{1} \mathrm{MD}$, Zeki ARI, PhD, \\ B. Sami UYANIK, MD, \\ and Hilal M. ÖzILGILI, MD
}

\section{SUMMARY}

Background. Studies have shown that patients with chronic renal failure have a high frcquency of cardiocascular atheromatous disease.

Methods. We examined serum lipoprotein (a) [Lp(a)], very-low density lipoprotein cholesterol (VLDL-C), low-density lipoprotein cholesterol (LDL-C) and high-density lipoprotein cholesterol (HDL-C), apolipoprotein Al (apo Al) and B (apo B), triglyceride (TG) and total cholesterol (TC) levels as possible risk factors for atherosclerosis in 45 patients with chronic renal failure (CRF) treated by hemodialysis (HD) and in 15 CRF patients who were not on HD. A control group of 20 healthy subjects was also studied.

Results. The proportion of smokers and body mass indexes were similar between the groups. In both patient groups, higher $\mathrm{TG}, \mathrm{TC}$ and $\mathrm{Lp}(\mathrm{a})$ and lower apo Al and HDL-C levels in serum were found than in those of controls. Serum apo B and LDL-C were similar in the patients treated by $\mathrm{HD}$ and the controls. Serum VLDL-C and LDL-C were similar in the CRF patients who were not on $\mathrm{HD}$ and the controls. The highest ratios of apo $\mathrm{B} / \mathrm{apo} \mathrm{Al}$ and LDL-C/HDL-C were found in HD patients. The highest ratio of TC/HDL-C was found in the other patient group.

We found significant correlations between $\mathrm{Lp}(\mathrm{a})$ and other parameters of lipoprotein metabolism in CRF patients, both those who were and those who were not on HD.

Conclusions. Our results indicate that CRF patients who both were and were not on HD show atherogenic changes in the lipoprotein pattern, and that the increase in $\mathrm{Lp}(\mathrm{a})$ during the CRF phase is basically related to the loss of renal function and may also depend on the resultant alterations which are produced in other lipoprotein variables. (Jpn Heart J 1997; 38; 83-89)

Key words: Apolipoproteins, Lipoprotein (a), Chronic renal failure and hemodialysis

From Department of Biochemistry, Medical School, Celal Bayar University, Manisa, Turkey and 'Department of Biochemistry, Medical School, Atatürk University, Erzurum, Turkey.

Address for correspondence: M. Ramazan Yiǧitoğlu, MD, Director of Clinical Laboratories \& Chief, Department of Biochemistry, Medical School, Gelal Bayar University, Manisa, Turkey.

Received for publication November 22, 1995.

Accepted July 17, 1996. 


\begin{abstract}
THEROSCLEROSIS is a multifactorial disease that encompasses both A genetic and environmental factors. Numerous epidemiological studies have shown that lipids/lipoproteins have a major role in the evolution of atherosclerosis. ${ }^{1)}$ Subjects with chronic renal failure (CRF) have a greatly increased risk of coronary heart disease and dislipidemia, and cerebrovascular and cardiovascular diseases are the major causes of death in maintenance hemodialysed patients with CRF. ${ }^{2,3)}$ Lipid abnormalities have been identified as significant risk factors for the development of premature atherosclerosis in such patients. These abnormalities include significant elevations of serum triglycerides, triglyceride-rich lipoproteins (VLDL, IDL, LDL remnant particles) and $\mathrm{Lp}(\mathrm{a})$ and decrease in HDL, accompanied by changes in apolipoprotein content. ${ }^{2)}$ However, little is known about the mechanisms involved in the lipid and lipoprotein abnormalities in paticnts with GRF treated by HD.

$\mathrm{Lp}(\mathrm{a})$ is a known risk factor for coronary heart disease in the general population. ${ }^{4-7)}$ Relatively few studies have examined the relationship of CRF and $\operatorname{Lp}(\mathrm{a})$ concentrations and investigated the relationship between $\operatorname{Lp}(\mathrm{a})$ and other lipid and lipoprotein concentrations in patients with CRF treated by HD. ${ }^{3,8,9)}$ The purpose of this study was to determine $\mathrm{Lp}(\mathrm{a})$ levels and to investigate the association of $\mathrm{Lp}(\mathrm{a})$ and atherosclerotic risk factors in patients with CRF, who were and were not on HD.
\end{abstract}

\title{
Methods
}

Subjects: The study was conducted in accordance with the ethical standards of the Research Hospital of Atatürk University, in Erzurum City, Turkey. We prospectively studied 45 subjects ( 25 men and 20 women) with CRF treated by HD, 15 patients with CRF who were not on HD (9 men, and 6 women) and 20 normal subjects as the reference group (11 men and 9 women). The proportion of smokers was similar among the groups. The patients were not receiving antilipemic drugs. Following a 12-hour fasting period, sample collection was carried out between $08: 00-10: 00 \mathrm{~h}$ both in control subjects and patients. Blood samples were withdrawn in hemodialysis patients at the start of the first dialysis session of the week. Blood was allowed to clot, and the serum was separated by centrifugation at $800 \mathrm{~g}$ for $15 \mathrm{~min}$. Serum aliquots were stored at $-20^{\circ} \mathrm{C}$ until determination of serum lipids and lipoproteins.

Renal function tests and lipid profiles: Serum BUN and creatinine levels were measured with the UV-kinetic and Jaffe's methods, respectively. ${ }^{10,11)} \mathrm{TC}$. and TG were determined enzymatically (Boehringer GmbH Mannheim, Mannheim, Germany) on a Mitsubishi Super Z 818 analyzer, (Mitsubishi, Tokyo, Japan). HDL-C was quantified by the same enzymatic method after 
precipitation of VLDL-G and LDL-G with phosphotungstic acid. LDL-G and VLDL-C were calculated according to the Friedewald equation: ${ }^{12)} \mathrm{LDL}-\mathrm{C}=\mathrm{TC}-$ (HDL-C + VLDL-C). This widely used formula is based on the assumption that the mass of VLDL-C present in serum is equivalent to one fifth of the mass of triglycerides in serum VLDL. ${ }^{13)}$

Serum $\operatorname{Lp}(\mathrm{a})$ concentrations were measured with a commercial ELISA (Boehringer Mannheim GmbH, Mannheim, Germany). Serum apo A-1 and apoB concentrations were measured by immunoturbidimetric analysis (Isolab Inc., Ohio, USA, Cat No 8253 and 8220 , respectively).

Statistical analysis: All results are expressed as mean \pm SD. Two-sample $t$-test was used to compare results for patients and controls. In order to analyse the relationships between parameters, correlation analysis was used. Day-to-day coefficients of variation (CVs) were $3 \%$ for assay of TC, $5.1 \%$ for HDL-C, $4.8 \%$ for TG, $5.6 \%$ for apo A-1, $4.2 \%$ for apo B, and $5.3 \%$ for $\mathrm{Lp}(\mathrm{a})$.

\section{RESULts}

All patients had increased serum BUN and creatinine, as shown in Table $\mathbf{I}$. Compared with controls, patients with CRF treated by HD had significantly higher amounts of TG, TC, VLDL-C, Lp (a), and lower apo A-1 and HDL-C in serum (Table II). Serum apo B and LDL-G werc similar in the paticnts who were on HD and the controls. The ratios of apo B/apo A-1, LDL-C/HDL-C, and TC/HDL-C werc higher in these patients than in the controls $(p<0.001) . \mathrm{Pa}-$ tients who were not on HD had higher serum TG, TC, Lp (a), and lower apo A1, apo B and HDL-G levels than the controls. Also, they had higher LDL-C/ HDL-C and TC/HDL-C ratios than the controls. However, there was no difference in the apo B/apo A-1 ratio between these patients and the controls.

The relationships between serum $\mathrm{Lp}(\mathrm{a})$ and other lipid parameters involved

Table I. Baseline Clinical and Laboratory Profiles of the Study Population

\begin{tabular}{lcccc}
\hline & Controls & \multicolumn{2}{c}{ Patients } & Statistical \\
& & CRF not on HD & CRF on HD & significance* \\
\hline Number of subjects & 20 & 15 & 45 & \\
Sex, M/F & $11 / 9$ & $9 / 6$ & $25 / 20$ & NS \\
Age, years & $44.9 \pm 6.5$ & $45 \pm 7.1$ & $42.2 \pm 12$ & NS \\
BMI $\left(\mathrm{kg} / \mathrm{m}^{2}\right)$ & $24.7 \pm 2.4$ & $23.5 \pm 2.84$ & $25.5 \pm 5$ & NS \\
MAP $(\mathrm{mmHg})$ & $105 \pm 21$ & $109 \pm 22$ & $108 \pm 24$ & $<0.001$ \\
BUN $(\mathrm{mg} / \mathrm{d} l)$ & $17.8 \pm 5.6$ & $64.6 \pm 15.3$ & $80.4 \pm 29.4$ & $<0.001$ \\
Creatinine $(\mathrm{mg} / \mathrm{d} l)$ & $1.03 \pm 0.18$ & $7.14 \pm 1.73$ & $8.25 \pm 3.9$ & \\
\hline
\end{tabular}

*Comparison between control group and patients who were and were not on hemodialysis, NS = not signif. cant; $\mathrm{MAP}=$ mean arterial pressure; $\mathrm{HD}=$ hemodialysis. 
Table X. Senum Lipid, Apolipoprotein, Lipoprotein Cholesterol and Lipoprotein (a) Levels in the Controls and Patients

\begin{tabular}{|c|c|c|c|c|c|c|}
\hline \multirow{2}{*}{ Parameters } & \multirow{2}{*}{ Controls } & \multicolumn{2}{|c|}{ Patients } & \multicolumn{3}{|c|}{ Statistical significance } \\
\hline & & CRF not on HD & CRF on HD & 1 & 2 & 3 \\
\hline \multicolumn{7}{|l|}{ Lipids, mg/d $l$} \\
\hline TG & $115 \pm 21.8$ & $137 \pm 32.6$ & $163 \pm 44$ & $<0.05$ & $<0.001$ & $<005$ \\
\hline $\mathrm{TC}$ & $145 \pm 31.5$ & $186 \pm 44.2$ & $188 \pm 85$ & $<0.01$ & $<0.05$ & NS \\
\hline \multicolumn{7}{|l|}{ Apolipoprotein, $\mathrm{mg} / \mathrm{d} l$} \\
\hline apo $\mathrm{Al}$ & $116 \pm 10.8$ & $100 \pm 21$ & $87 \pm 19$ & $<0.01$ & $<0.001$ & $<0.05$ \\
\hline apo $B$ & $98.9 \pm 13.7$ & $87.5 \pm 15.7$ & $114 \pm 33$ & $<0.05$ & NS & $<0.01$ \\
\hline \multicolumn{7}{|c|}{ Lipoprotein cholesterol, $\mathrm{mg} / \mathrm{d} l$} \\
\hline VLDL & $24.9 \pm 3.7$ & $26.4 \pm 8.2$ & $32.3 \pm 9$ & NS & $<0.01$ & $<0.05$ \\
\hline LDL & $96.5 \pm 22.3$ & $88 \pm 23.4$ & $123 \pm 87$ & NS & NS & NS \\
\hline HDL & $41.8 \pm 8.2$ & $31.9 \pm 5.6$ & $33.2 \pm 11$ & $<0.001$ & $<0.01$ & NS \\
\hline Lipoprotein (a), mg/dl & $19.4 \pm 4.9$ & $26.8 \pm 5.2$ & $29.7 \pm 19.5$ & $<0.01$ & $<0.01$ & NS \\
\hline apo $\mathrm{B}$ /apo $\mathrm{Al}$ & $0.86 \pm 0.13$ & $0.92 \pm 0.28$ & $1.33 \pm 0.59$ & NS & $<0.001$ & NS \\
\hline LDL-C/HDL-C & $2.31 \pm 0.30$ & $3.09 \pm 0.97$ & $3.69 \pm 0.91$ & $<0.01$ & $<0.001$ & $<0.05$ \\
\hline TC/HDL-C & $3.53 \pm 0.78$ & $6.03 \pm 1.87$ & $5.68 \pm 1.46$ & $<0.001$ & $<0.001$ & NS \\
\hline
\end{tabular}

1: Comparison between control group and patient group which was not on HD; 2: Comparison between control group and patient group on HD; 3 : Comparison among the two patient groups which was or was not on $\mathrm{HD}$.

Table III. Correlation Coefficients (r) between $\mathrm{Lp}(\mathrm{a})$ and Other Parameters in Controls and Patients

\begin{tabular}{lccc}
\hline \multirow{2}{*}{ Parameters } & Control Lp (a) & \multicolumn{2}{c}{ Patient Lp (a) } \\
\cline { 3 - 4 } & & CRF not on HD & CRF on HD \\
\hline TG & $-0.18^{\mathrm{NS}}$ & $0.73^{* *}$ & $0.52^{* *}$ \\
TC & $0.33^{\mathrm{NS}}$ & $0.76^{* *}$ & $0.87^{* *}$ \\
HDL-C & $-0.08^{\mathrm{NS}}$ & $-0.60^{* *}$ & $-0.55^{* *}$ \\
LDL-C & $-0.28^{\mathrm{NS}}$ & $0.75^{* *}$ & $0.87^{* *}$ \\
VLDL-C & $0.19^{\mathrm{NS}}$ & $0.47^{*}$ & $0.52^{* *}$ \\
apo Al & $0.12^{\mathrm{NS}}$ & $-0.52^{* *}$ & $-0.40^{*}$ \\
apo B & $0.09^{\mathrm{NS}}$ & $0.54^{*}$ & $0.59^{* *}$ \\
\hline
\end{tabular}

Ns $=$ not significant; $*=$ significant at $p<0.05$ and $* *=p<0.001$

in lipids of patients and controls were also studied (Table III). In patient groups, serum L.p(a) levels were negatively correlated with serum HDL-C and apo A-1 levels, and positively correlated with TG, TC, LDL-C, VLDL-C, and apo B. In the controls, there were no significant relationships between serum $\mathrm{Lp}(\mathrm{a})$ and other lipid, lipoprotein, and apolipoprotein levels.

\section{Discussion}

Our study conclusively demonstrates lipoprotein and lipid abnormalities associated with CRF in patients both on and off $\mathrm{HD}$. The concentrations of TG and TC in serum were higher in both patient groups than those in the controls, 
and apo A-1 and HDL-C levels were lower in patients. These findings confirm previous reports in the literature. Roh $^{12)}$ reported that atherogenesis accelerates in HD patients. Cuxart et al ${ }^{14)}$ detected hypertriglyceridemia in $34 \%$ of $\mathrm{HD}$ patients. In the present study, the prevalence of hypertriglyceridemia (greater than $200 \mathrm{mg} / \mathrm{d} l$ ) was $20 \%$ and $16 \%$ in patients who were not on HD and were on HD, respectively. Schumaker et al. ${ }^{15)}$ reported increased concentrations of serum TG and apo B in patients with CRF. Haaber et al. ${ }^{16)}$ found that the plasma concentrations of lipoproteins showed an atherogenic pattern in CRF patients with increased levels of VLDL-C and TG, but a decreased level of HDLC. According to the study of Kaysen, ${ }^{177}$ plasma TG is increased in the majority of patients with advanced renal failure but cholesterol is not. He also reported that the HDL-associated apolipoproteins apo A-1 and A-II are decreased, while apo $B$ is increased in these patients. Segerra et al. ${ }^{97}$ reported that patients with CRF had lower serum levels of HDL-C and apo A-1, and higher TG and VLDL-C than did the control group. In the present study, apo B and LDL-C were similar in the patients treated by HD and controls.

Lipoprotein catabolic rate is reduced in patients with CRF, but the cause of hyperlipidemia is multifactorial; e.g. reduced lipoprotein lipase activity (LPL), increased concentration of apo C-III (a specific inhibitor of LPL) in plasma, secondary hyperparathyroidism, and insulin resistance. ${ }^{17-19)}$ Hörkkö et al. ${ }^{20)}$ reported that the clearance of $\mathrm{LDL}$ in patients with advanced uremia on conservative management is frequently decreased. These alterations in the metabolism of the most atherogenic particle in plasma or serum may contribute to the accelerated atherosclerosis in uremic patients. While atherosclerotic disease and thrombosis are the leading causes of death in dialysis patients, it is not certain that the specific lipid disorders of CRF are responsible for this morbidity, nor is it recommended at this time that qualitative abnormalities be treated pharmacologically in the absence of increased lipid levels. ${ }^{17,20)}$ When hyperlipidemia is present, plasma newly-synthesized cholesteryl ester transfer rates are raised and may contribute to elevated levels of the proatherogenic apo B-containing lipoproteins in patients with renal failure. ${ }^{21)}$

Querfeld et al. ${ }^{22)}$ found that the distribution of $\mathrm{Lp}(\mathrm{a})$ levels in normal subjects was skewed to the left with $97 \%$ having levels less than $30 \mathrm{mg} / \mathrm{dl}$. All normal subjects in this study had serum $\mathrm{Lp}(\mathrm{a})$ levels below $30 \mathrm{mg} / \mathrm{d} l$. Cuxart et al. ${ }^{14)}$ showed a higher prevalence of positive $\mathrm{Lp}$ (a) (greater than $30 \mathrm{mg} / \mathrm{d}$ ) in the HD group. The number of high-Lp(a) level patients on HD was $14(31 \%)$ in this study.

Cressman et al $^{23)}$ found that patients on HD have four-fold higher $\operatorname{Lp}(\mathrm{a})$ levels in serum than those of controls. They suggested that dialysis has a negative effect on lipid metabolism. 
In this study, the ratios of apo B/apo A-1, LDL-C/HDL-C, and TC/HDL$\mathrm{C}$ were higher in patients with $\mathrm{CRF}$ on $\mathrm{HD}$ as compared with controls. Also, patients who were not on HD had higher ratios of LDL-C/HDL-G and TC/ HDL-C. However, the ratio of apo B/apo A-1 in these patients was not different than that of the controls. We could not find similar reports in terms of these parameters in the literature.

There were significant correlations between $\mathrm{Lp}(\mathrm{a})$ and other parameters of lipid and lipoprotein metabolism in patients with CRF who were or not on HD in the present study. However, conflicting results about these relationships have been reported in previous studies. ${ }^{8,9,24-26)}$ Segerra et al..$^{9)}$ reported that there was no relationship between $\operatorname{Lp}(\mathrm{a})$ and other parameters of lipoprotein metabolism, for example, apolipoproteins A1, B, C-II, and C-III, HDL-C and LDL-C in patients with CRF before renal transplantation. Thus, they concluded that the increase in $\mathrm{Lp}(\mathrm{a})$ during the CRF phase is basically related to the loss of renal function and does not depend on the resultant alterations which are produced in other lipoprotein variables. Takegoshi et al. ${ }^{25)}$ found a statistically significant correlation of $\mathrm{Lp}(\mathrm{a})$ with serum lipoprotein and apolipoprotein levels in patients with CRF treated by continuous ambulatory peritoneal dialysis (CAPD). Hoffner et al. ${ }^{8)}$ reported that $\operatorname{Lp}(\mathrm{a})$ levels were not correlated with the level of creatinine in subjects with CRF. Thus, the elevation of $\mathrm{Lp}(\mathrm{a})$ levels in renal failure must occur early in renal failure, or alternatively, elevated $L p(a)$ levels may promote progression to CRF. However, the cause of the controversial results may stem from the different study groups in our study and the above studies. For example, Kandoussi et al. ${ }^{26)}$ reported that plasma Lp(a) level was high in predialysis or HD, but not in CAPD, as compared to healthy controls, though Takegoshi et al. ${ }^{25)}$ found high $\mathrm{Lp}(\mathrm{a})$ levels in CAPD patients. Hörkkö et al ${ }^{20)}$ showed that the $\mathrm{Lp}(\mathrm{a})$ levels were related to the plasma TC $(r=0.69)$, VLDL-C $(r=0.86)$, and LDL-G $(r=0.66)$ in patients with CRF. These results are consistent with our results.

Our results indicate that increased serum $\mathrm{Lp}(\mathrm{a})$ together with other lipoprotein abnormalities in patients with CRF who were and were not on HD imply an increased cardiovascular risk, and that there were significant relationships between $\mathrm{Lp}(\mathrm{a})$ and other parameters of lipoprotein metabolism such as TG, TC, HDL-C, LDL-C, VLDL-C, apo A-1 and apo B.

\section{References}

1. Roheim PS, Asztalos BF. Clinical significance of lipoprotein size and risk of coronary atherosclerosis. Clin Chem 1995; 41: 147-52.

2. Musidl J, Undas A, Syslo K. Lipid metabolism abnormalities and atherosclerosis in patients on maintenance hemodialysis. Przegl Lek 1994; $11: 264-7$. 
3. Ishikawa $\mathbf{H}$. Lipoprotein (a) is a risk factor for cerebrovascular accident in patients with renal failure. Nippon-Jinzo-Gakkai-Shi 1994; 36: 846-52 (in Japanese).

4. Scanu AM. Lipoprotein (a): a potential bridge between the fields of atherosclerosos and thrombosis. Arch Pathol Lab Med 1988; 112: 1405-7.

5. Scanu AM. Lipoprotein (a): a genetic risk factor for premature coronary heart disease. JAMA 1993; 267: 3326-9.

6. Scanu AM, Lawn R, Berg K. Lipoprotein (a) and atherosclerosis: Davis Conference. Ann Intern Med 1991; 115: 209-18.

7. Ridken PM, Hennekens CH, Stampfer MJ.A. Prospective study of lipoprotein (a) and risk of myocardial infarction. JAMA 1993; 270: 2195-225.

8. Haffner SM, Gruber KK, Aldrete G, Morales PA, Stern MP, Tuttle KR. Increased lipoprotein (a) concentrations in chronic renal failure. J Am Soc Nephrol 1992; 3: 1156-62.

9. Segarra A, Chacon P, Martin M, et al. Serum lipoprotein (a) levels in patients with chronic renal failure-evolution after renal transplantation and relationship with other parameters of lipoprotein metabolism: a prospective study. Nephron 1995; 69: 9-13.

10. Sampson EJ, Baird MA, Burtis CA. Coupled-enzyme equilibrium method for measuring urea in serum: optimization and evaluation of AACC study group on urea candidate reference method. Clin Chem 1980; 26: 816 26.

11. Buttler AR. The Jaffe reaction: identification of the colored species. Clin Chirn Acta 1976; 59: 229-32.

12. Roh DD. Is atherogenesis accelerated in hemodialysis patients? Int J Artificial Organs 1992; 15: 3236.

13. Li KM, Wilcken DE, Dudman NPB. Effect of serum lipoprotein (a) on estimation of low-density lipoprotein cholesterol by the Friedewald formula. Clin Chem 1994; 40: 571-3.

14. Cuxart M, Sans R, Vicente M, Perez-Gallofre A, Masdeu-Jufre S, Ponsati C. Lipoprotein $\operatorname{Lp}(\mathbf{a})$ in patients with chronic renal insufficiency on hemodialysis. Med Clin Barc 1989; 92: 769-71 (in Spanish).

15. Schumaker M, Keßler A, Bahlmann G, Wood WG. Acute changes in concentrations of apolipoproteins A-1, B, C-II and lipoprotein (a) in serum covering the period from directly before to 48 hours after chronic haemodialysis. Eur J Clin Chem Clin Biochem 1994; 32: 123-5.

16. Haaber $A B$, Eidemak I, Jensen $T$, Feldt RB, Strandgaard $S$. Vascular endothelial cell function and cardiovascular risk factors in patients with chronic renal failure. J Am Soc Nephrol 1995; 5: 1581-4.

17. Kaysen GA. Hyperlipidemia of chronic renal failure. Blood Purif 1994; 12: 60-7.

18. Attman PO, Samuelsson O, Alaupovic P. Lipoprotein metabolism and renal failure. Am J of Kidney Disease 1993; 21: 573-92.

19. Attman PO, Alaupovic P. Lipid abnormalities in chronic renal insuficiency. Kidney Int 1991; 39 (Supp 31): 16-23.

20. Hörkkö S, Huttunen K, Korhonen T, Kesaniemi YA. Decreased clearance of low-density lipoprotein in patients with chronic renal failure. Kidney Int 1994; 45: 561-70.

21. Corboy J, Sutherland WH, Walker RJ, Robertson MC, Cox CM. Cholesteryl ester transfer in patients with renal failure or renal transplants. Kidney Int 1994; 46: 1147-53.

22. Querfeld U, Lang M, Friedrich JB, Kohl B, Fiehn W, Scharer K. Lipoprotein(a) serum levels and apolipoprotein (a) phenotypes in children with chronic renal disease. Pediatr Res 1993; 34; 722-6.

23. Cressman MD, Heyka RJ, Paganini EP, O'Neil J, Skibinski CL, Hoff HF. Lipoprotein (a) is an independent risk factor for cardiovascular disease in hemodialysis patients. Circulation 1992; 86: 47582.

24. Okura Y, Saku K, Hirata K. Serum lipoprotein(a) levels in maintenance hemodialysis patients. Nephron 1993; 65: 46-50.

25. Takegoshi T, Kitoh C, Shimada T, Kawai K, Yamazaki Y, Mabuchi H. Alterations of Lp (a) lipoprotein in patients with chronic renal failure treated by continuous ambulatory peritoneal dialysis. NipponJinzo-Gakkai-Shi 1993; 35: 757-63 (in Japanese).

26. Kandoussi A, Cachera C, Pagniez D, Dracon M, Fruchart JC, Tacquet A. Plasma level of lipoprotein Lp (a) is high in predialysis or hemodialysis, but not in CAPD. Kidney Int 1992; 42: 424-5. 